\title{
Recipe Development and Mechanical Characterization of Carbon Fibre Reinforced Recycled Polypropylene 3D Printing Filament
}

\author{
Mwambe Polline', James M. Mutua², Thomas Ochuku Mbuya3 ${ }^{3}$, Kyekyere Ernest ${ }^{1}$ \\ ${ }^{1}$ Department of Mechanical Engineering, Pan African University Institute for Basic Sciences, Technology and Innovation, Nairobi, \\ Kenya \\ ${ }^{2}$ Department of Mechanical Engineering, Jomo Kenyatta University of Agriculture and Technology, Nairobi, Kenya \\ ${ }^{3}$ Department of Mechanical \& Manufacturing Engineering, University of Nairobi, Nairobi, Kenya \\ Email: mwambepauline@gmail.com
}

How to cite this paper: Polline, M., Mutua, J.M., Mbuya, T.O. and Ernest, K. (2021) Recipe Development and Mechanical Characterization of Carbon Fibre Reinforced Recycled Polypropylene 3D Printing Filament. Open Journal of Composite Materials, 11, 47-61.

https://doi.org/10.4236/ojcm.2021.113005

Received: June 27, 2021

Accepted: July 27, 2021

Published: July 30, 2021

Copyright (c) 2021 by author(s) and Scientific Research Publishing Inc. This work is licensed under the Creative Commons Attribution International License (CC BY 4.0).

http://creativecommons.org/licenses/by/4.0/

\begin{abstract}
Recycled polypropylene filaments for fused filament fabrication were investigated with and without $14 \mathrm{wt} \%$ short fibre carbon reinforcements. The microstructure and mechanical properties of the filaments and 3D printed specimens were characterized using scanning electron microscopy and standard tensile testing. It was observed that recycled polypropylene filaments with 14 $\mathrm{wt} \%$ short carbon fibre reinforcement contained pores that were dispersed throughout the microstructure of the filament. A two-stage filament extrusion process was observed to improve the spatial distribution of carbon fibre reinforcement but did not reduce the pores. Recycled polypropylene filaments without reinforcement extruded at high screw speeds above $20 \mathrm{rpm}$ contained a centreline cavity but no spatially distributed pores. However, this cavity is eliminated when extrusion is carried out at screw speeds below 20 rpm. For 3D printed specimens, interlayer cavities were observed larger for specimens printed from $14 \mathrm{wt} \%$ carbon fibre reinforced recycled polypropylene than those printed from unreinforced filaments. The values of tensile strength for the filaments were $21.82 \mathrm{MPa}$ and $24.22 \mathrm{MPa}$, which reduced to 19.72 $\mathrm{MPa}$ and 22.70 MPa, respectively, for 3D printed samples using the filaments. Likewise, the young's modulus of the filaments was $1208.6 \mathrm{MPa}$ and 1412.7 $\mathrm{MPa}$, which reduced to $961.5 \mathrm{MPa}$ and 1352.3 $\mathrm{MPa}$, respectively, for the $3 \mathrm{D}$ printed samples. The percentage elongation at failure for the recycled polypropylene filament was $9.83 \%$ but reduced to $3.84 \%$ for the samples printed with $14 \mathrm{wt} \%$ carbon fiber reinforced polypropylene filaments whose elongation to failure was $6.58 \%$. The SEM observations on the fractured tensile test samples showed interlayer gaps between the printed and the adjacent raster layers. These gaps accounted for the reduction in the mechanical prop-
\end{abstract}


erties of the printed parts.

\section{Keywords}

Fused Filament Fabrication, Fused Deposition Modeling, 3D Printing, Carbon Fibre Reinforced Polymers, Polypropylene, Plastic Recycling

\section{Introduction}

$3 \mathrm{D}$ printing is an additive manufacturing process that produces components from 3D CAD files through added material layers [1] [2]. It allows physically tangible products drawn in CAD software to be fabricated by depositing materials layer by layer. This new industrial revolutionary process has shown rapid adoption and growth in product development and manufacturing. The process uses a range of rapid prototyping methods, including fused deposition modeling, powder-bed printing, ink-jetting, stereolithography, selective laser sintering, and many other new methods that have been developed for 3D printing of composites [3]. Among these methods, fused deposition modeling (FDM) or fused filament fabrication (FFF) is the predominant process used across manufacturing industries to make thermoplastic prototypes for functional testing [4] [5].

$\mathrm{FDM}$ or FFF is a $3 \mathrm{D}$ CAD-controlled process that melts the feedstock in the extrusion head [6]. The semi-molten material is extruded through a heated nozzle and deposited layer-by-layer to build a 3D part, as shown in Figure 1. Post-processing of the printed part may be necessary to clean and remove any support. In the FDM method, the first stage is to create a 3D model with CAD software. The part file is then transformed into a Stereolithography file (STL) which contains the instruction codes for the machine tool to pursue a defined tool path [7]. FDM technology offers lower cost, robustness, multi-material flexibility, and simplicity [4].

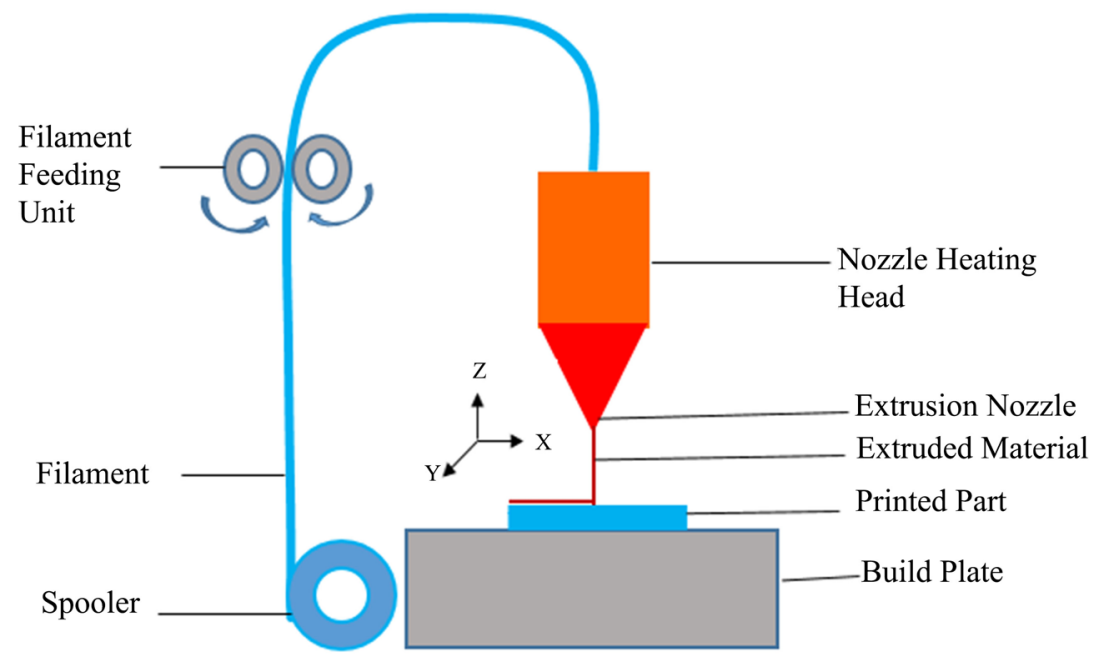

Figure 1. Schematic representation of the FDM or FFF process. 
Manufacturers develop products that exhibit multi-property characteristics through FDM filaments that are produced by heating, extruding, and cooling materials such as plastics to transform nurdles into the finished filaments. The FDM material filaments allow manufacturers to produce actual parts that can be used for prototyping, functional testing, installation and, most significantly, end-use [5]. Research in FDM shows that the primary materials used include acrylonitrile butadiene styrene (ABS), polyamide, polycarbonate, polyethylene (PE), and polypropylene (PP) [5] [8]. Busch et al. [9] characterized the five different materials used for 3D printing in fused deposition modeling, which included Acrylonitrile butadiene styrene (ABS), Polylactic acid (PLA), Nylon, Bendlay, and High Impact Polystyrene Sheet (HIPS).

Research shows that the open-source 3D printing platform rapidly expands the broader adoption of after-consumer plastic recycling at home [10] [11]. These studies reflect a possible evolution of a networked manufacturing future suitable for developed and developing countries with lower environmental impacts than the current system. In addition, the growth of desktop 3D printers has stimulated recycled 3D printer filament interest to reduce distributed production costs [12]. Seven types of plastics that are widely recycled today include polyethylene terephthalate (PET), high-density polyethylene (HDPE), polyvinyl chloride (PVC), low-density polyethylene (LDPE), polypropylene, polystyrene (PS), and primarily polycarbonate (PC) [12]. Although all these plastics are recyclable, few have been investigated and used to make filaments for 3D printing.

The growing adoption of 3D printing technology has enabled the use of advanced filament material, ranging from virgin pellets (ABS/PLA), plastic wastes, and recycled 3D printed composites [12]. Polypropylene plastic wastes have also been recycled for filament fabrication and use in FDM. Spoerk et al. [13] investigated the mechanical recyclability of PP-heat stabilized filaments for FDM applications suitable for multiple remanufacturing sequences. The study reported that PP composite in the course of 15 consecutive filament extrusions and additively manufactured PP composite revealed an unaltered morphology, same tensile and impact strength as the initial material. Zander et al. [14] also investigated the filament fabrication using recycled PP and PET wastes blends for 3D printing. The effect of blend composition and compatibilizer on the resulting mechanical properties showed that blends of PP/PET compatibilizer with styrene-ethylene/butylene-styrene (SEBS) and maleic anhydride functionalized SEBS had tensile strengths of $23 \pm 1 \mathrm{MPa}$ and $24 \pm 1 \mathrm{MPa}$, respectively. However, several 3D printed polymer components are still used as theoretical prototypes rather than practical components, primarily because these thermoplastic products lack the required strength and reliability as fully functional components [15]. The current development efforts in 3D printing of polymer composites contributes to the solution of these challenges by integrating fibre or nano-material reinforcements into polymers resulting in high mechanical strength and superior functionality [4].

Recent studies on the effect of adding carbon fibre of different content and 
length showed a trend in enhanced mechanical properties of the manufactured components [16] [17]. Ning et al. [18] studied the effect of adding carbon fibre to plastic materials. The study found that the carbon fibre increased the tensile strength and Young's modulus but decreased toughness, yield strength, and ductility. Mori et al. [19] also reported that the addition of carbon fibre reinforcement to ABS significantly increased the tensile strength and hardness of $3 \mathrm{D}$ printed parts. Therefore, there is a significant potential for improving the properties of FDM manufactured parts through the appropriate addition of reinforcements to polymer filament material. This improvement is expected to be more significant with the increased adoption of recycled thermoplastics to produce FDM filaments.

This paper, therefore, presents the results of an experimental study to develop high-quality carbon fiber reinforced recycled polypropylene (CFR-PP) filaments for FDM component manufacture. The ratios of carbon fiber reinforcements to the plastic waste matrix (CFR-PP composite mix) for the optimum mechanical properties were first established. Then the filament was extruded following the developed CFR-PP composite mix and used to produce 3D printed specimens. Mechanical testing of the filaments and 3D printed tensile test samples was carried out. The microstructure of the CFR-PP composite filaments and the fracture surface characteristics of failed tensile specimens were also investigated.

\section{Materials \& Methods}

\subsection{Filament Materials}

Granulated pellets of recycled PP (supplied by Mr. Green Africa, Nairobi, Kenya) of melt flow rate $33.8 \mathrm{~g} / 10 \mathrm{~min}$ (with $5000 \mathrm{~g}$ weight at $230^{\circ} \mathrm{C}$ ) and short carbon fibre (supplied by Shenzhen Yataida High-Tech. Co., Ltd, Guangdong, China) were used for the experimental study. The specifications of materials used are summarized in Table 1 as obtained from the suppliers.

\subsection{CFR-PP Filament Composition Design}

The determination of the optimum CFR-PP composite mix was a prerequisite for the extrusion of carbon fibre-reinforced recycled PP filament. Using the synthesizer tool in CES Edupack 2019 software, the percentage weight (wt\%) of the short carbon fibre was varied up to $20 \%$ using $1 \%$ increments. The effect of addition to two types of short carbon fibres (high strength and high modulus) to the PP polymer matrix was simulated. The simulation runs gave predicted physical and mechanical properties of the resulting composite (e.g. density, Young's

Table 1. Specifications of filament materials.

\begin{tabular}{cccccc}
\hline Material & $\begin{array}{c}\text { Density } \\
\left(\mathrm{g} / \mathrm{cm}^{3}\right)\end{array}$ & $\begin{array}{c}\text { Tensile } \\
\text { Strength }(\mathrm{MPa})\end{array}$ & $\begin{array}{c}\text { Young's } \\
\text { Modulus (GPa) }\end{array}$ & $\begin{array}{c}\text { Average fiber } \\
\text { length (um) }\end{array}$ & $\begin{array}{c}\text { Fibre/Pellet } \\
\text { diameter }\end{array}$ \\
\hline Recycled PP & 0.574 & 22.5 & 1.24 & - & $5.04 \mathrm{~mm}$ \\
Short carbon fiber & 1.75 & 3500 & 230 & 200 & $7.0 \mathrm{um}$ \\
\hline
\end{tabular}


Modulus, tensile strength). The results showed an increasing trend of the composite filament's physical and mechanical properties at the expense of flexibility. The flexibility of the filament is a crucial requirement for feedstock winding on the spooler.

To achieve optimum values of desired properties and maintain filament flexibility, a design of experiment (DOE) was performed using Minitab 19 software. The two input factors (high strength and high modulus short carbon fibre) and 20 levels of the $1 \%$ incremental percentage weight (wt\%) of the short carbon fibre were modeled using the general full factorial design. The test order was randomized, and all the points were replicated once, which resulted in 40 experimental combinations. The output factors were density, the young's modulus, and tensile strength. Finally, with a significance level of 5\%, the individual targeted values of desired mechanical properties for a specific material application were combined, and a $14 \mathrm{wt} \%$ high modulus short carbon fibre composition was found to predict the most improved properties. The detailed results are given in the result section.

\subsection{Filament Fabrication}

The single-screw extruder machine (SJ35, Zhangjiagang, China) that feeds the raw material into the three controlled heating zones with a die nozzle of $3.0 \mathrm{~mm}$ diameter was used to extrude filaments of both recycled PP and CFR-recycled $\mathrm{PP}$. The barrel and die nozzle zones were first preheated to a set temperature of $170^{\circ} \mathrm{C}$ and $180^{\circ} \mathrm{C}$. This preheating is necessary before the materials are fed through the hopper. Simultaneously, the barrel screw speed was set at $10 \mathrm{rpm}$ forward rotation to push melted material up to the die nozzle. The feed cooling zone was also set to a temperature of $60^{\circ} \mathrm{C}$ to preheat the pellets/mixture before entering the barrel zone. For each feeding time, a pre-weighed $300 \mathrm{~g}$ of granulated PP pellets and 14\% wt carbon (300 g of granulated PP pellets mixed adequately with $42 \mathrm{~g}$ of short carbon fiber) were fed into the barrel when the preset temperatures were reached for the fabrication of recycled PP and CFR-recycled PP filaments. The hot extruded filament was then pulled off by the filament reducer rollers set at a speed of $200 \mathrm{~mm} / \mathrm{s}$ through a water-cooled bath. Finally, the extruded filament was wound up and spooled on the spooler rotating at a speed of $100 \mathrm{~mm} / \mathrm{s}$ with the help of filament storage rollers. The filament extrusion processing parameters were adjusted accordingly, as summarized in Table 2, to maintain acceptable filament diameter ranges.

\subsection{Specimen Manufacture Using Fused Filament Fabrication (FFF)}

The fabricated filaments were used for 3D printing using an Ultimaker S3 (Ultimaker, Zaltbommel, Netherlands). Ultimaker Cura 4.8.0 open-source software was used to generate the stl files for the printing process. The CAD model of the standard tensile test specimen (Type 1, ASTM D638 [20]), was drawn using Solidworks 2020 software (Figure 2). Five tensile test specimens were then printed 
Table 2. Filament extrusion process parameters.

\begin{tabular}{ccc}
\hline Type of material & Recycled PP & CFR-recycled PP \\
\cline { 2 - 3 } Parameters & Value & Value \\
\hline Barrel Temp $\left({ }^{\circ} \mathrm{C}\right)$ & 170 & 180 \\
Die/nozzle Temp $\left({ }^{\circ} \mathrm{C}\right)$ & 180 & 190 \\
Screw extrusion speed $(\mathrm{rpm})$ & $18-20$ & $13-15$ \\
Filament pulling roller speed $(\mathrm{mm} / \mathrm{s})$ & 300 & 250 \\
Filament winding roller speed $(\mathrm{mm} / \mathrm{s})$ & 200 & 150 \\
\hline
\end{tabular}

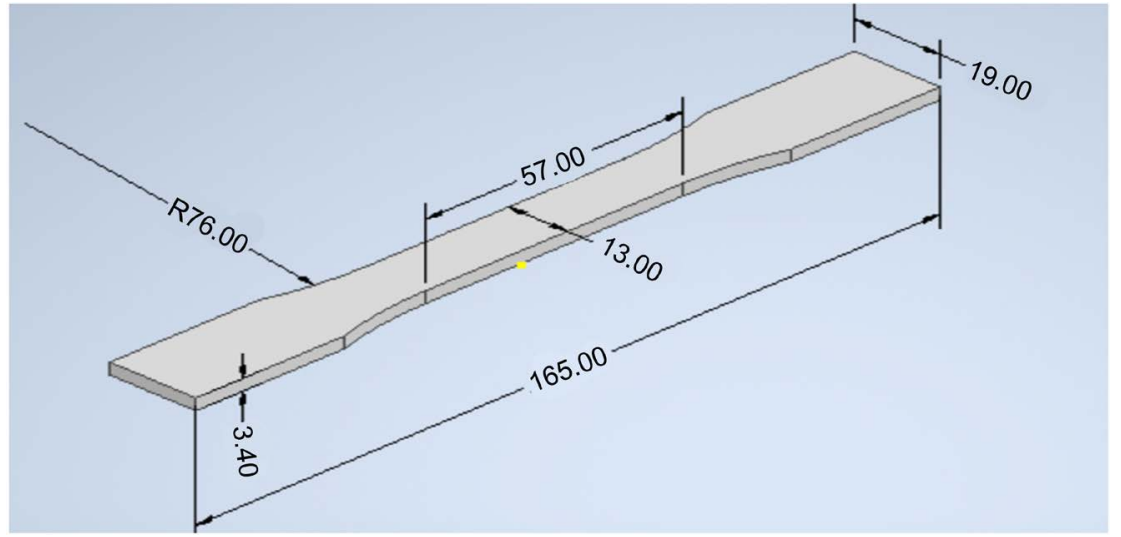

Figure 2. Tensile test specimen dimensions.

using the recycled PP and CFR-recycled PP filaments. Printing nozzle diameters of $0.4 \mathrm{~mm}$ and $0.6 \mathrm{~mm}$ were used for filaments of recycled PP and CFR-recycled PP, respectively. The printing process parameters used are shown in Table 3, including printing strategy of $[0,90]$ degrees and percentage infill of $100 \%$.

The PP thermoplastics used for the extrusion of filaments were from recycled common commercial grade PP, prone to shrinkage and warpage upon cooling during $3 \mathrm{D}$ printing [21]. There was challenge of adhesion of the first $3 \mathrm{D}$ printed specimen layer onto the printer build plate. This is the main reason why most commercially marketed PP filaments are modified with blends and composites to overcome this warpage problem [22]. While using Pritt glue and Kapton tape, there was no adhesion of the first print layer onto the printer bed and resulted in the premature termination of the printing. The use of packing tape (Sumo Tape) ensured sufficient adhesion of the print onto the printing bed throughout the printing process. Additionally, the selection of brim adhesion type compared to raft delivered better printing results and compensated for warpage.

\subsection{Tensile Testing}

Tensile testing was carried out for both the extruded filaments and 3D printed specimens. The tests were conducted under ambient conditions with a gauge length of $50 \mathrm{~mm}$ for the filaments and $57.0 \mathrm{~mm}$ for 3D printed specimens. Tensile testing was carried out using a $10 \mathrm{kN}$ capacity tensile machine (ST series, Tinius Olsen, United States of America) at a constant loading speed of $50 \mathrm{~mm} / \mathrm{min}$. A 
Table 3. FFF printing parameters for recycled PP and CFR-recycled PP.

\begin{tabular}{ccc}
\hline Parameter & Recycled PP & CFR-recycled PP \\
\cline { 2 - 3 } & Values & Values \\
\hline Printing temperature $\left({ }^{\circ} \mathrm{C}\right)$ & 190 & 210 \\
Printing temperature, initial layer $\left({ }^{\circ} \mathrm{C}\right)$ & 200 & 220 \\
Build plate temperature $\left({ }^{\circ} \mathrm{C}\right)$ & 85 & 85 \\
Build plate temperature, initial layer $\left({ }^{\circ} \mathrm{C}\right)$ & 95 & 95 \\
Infill pattern & lines & lines \\
Infill flow $(\%)$ & 110 & 110 \\
Layer height $(\mathrm{mm})$ & 0.3 & 0.3 \\
Line width $(\mathrm{mm})$ & 0.38 & 0.38 \\
Top and bottom layers $($ layers $)$ & 2 & 2 \\
Print speed (mm/s) & 25 & 25 \\
Initial layer speed $(\mathrm{mm} / \mathrm{s})$ & 15 & 15 \\
Build plate adhesion type & brim & brim \\
\hline
\end{tabular}

desktop computer loaded with Horizon Software and connected to the tensile test machine was used to record load $(\mathrm{N})$ and displacement $(\mathrm{mm})$ measurements. This data was subsequently analyzed to obtain typical tensile stress-strain graphs that were then used to obtain tensile strength, elongation to fracture, and Young's Modulus. The fractured tensile specimens were carefully stored to preserve the integrity of the fracture surface for subsequent fractography as described in Section 2.6.

\subsection{Microstructure Characterization and Fracture Surface Analysis}

The microstructure of the extruded filaments and the 3D printed specimens were investigated using an environmental Scanning Electron Microscope (NeoScope JCM-7000, JEOL Tokyo) set at a low vacuum and an accelerating voltage of $10 \mathrm{kV}$. First, the extruded filaments were sectioned carefully through the cross-section using a razor blade. For SEM imaging, no special surface treatment or polishing was carried out on the samples. They were then mounted on a specimen holder using double-sided carbon sticker tape and inserted in SEM chamber for imaging.

Fractured tensile specimens were also sectioned at $2.0 \mathrm{~mm}$ from the fracture region to obtain a convenient specimen size for SEM observation. These were then mounted on a specimen holder using double-sided carbon sticker tape and inserted in SEM chamber for imaging. No special surface treatment was required for SEM imaging.

\section{Results and Discussion}

\subsection{CFR-PP Filament Composite Design Simulation Results}

The values of simulated results of predicted physical and mechanical properties 
of the composite filament in varying composition up to $20 \mathrm{wt} \%$ are shown in Figure 3. From the simulation results, the effect of adding high strength and high modulus short carbon fibres of weight percentage $(1 \%-20 \%)$ to the PP thermoplastic resulted in increasing properties of density, tensile strength, and the Young's. The increase in the density property for both high modulus-PP composite and high strength-PP composite was the same, majorly due to the same inherent value of density for the two types of carbon fibres. Tensile strength for high strength-PP composite was slightly higher than that of high modulus-PP composite, while the value for Young's Modulus was slightly lower than the counterpart. This phenomenon can be attributed to the high stiffness value of high strength short carbon fibres, making them rigid to any deformations.

Likewise, the simulated results of predicted physical and mechanical properties of the high modulus short CFR-PP composite in varying composition up to $20 \mathrm{wt} \%$ are shown in Figure 4. The simulated mechanical properties for $14 \mathrm{wt} \%$ high modulus CFR composite and the actual experimental results of CFR-Recycled PP filament are compared in Table 4.

\subsection{Filament Fabrication Using Recycled PP and CFR-Recycled PP Material}

The fabrication of filaments within acceptable Ultimaker S3 printer tolerance of $2.85 \mathrm{~mm}( \pm 0.05)$ is necessary for achieving constant filament feed during the additive manufacturing process. Constant filament feed during printing is achieved if the cross-sectional area of the filament is uniform. Preliminary trial printing showed that filaments of $2.0-3.0 \mathrm{~mm}$ diameter could be printed. To fabricate filaments within this acceptable printer range, the hot strands of filament extruded from the $3.0 \mathrm{~mm}$ diameter die were pulled by a controlled speed of the reducer
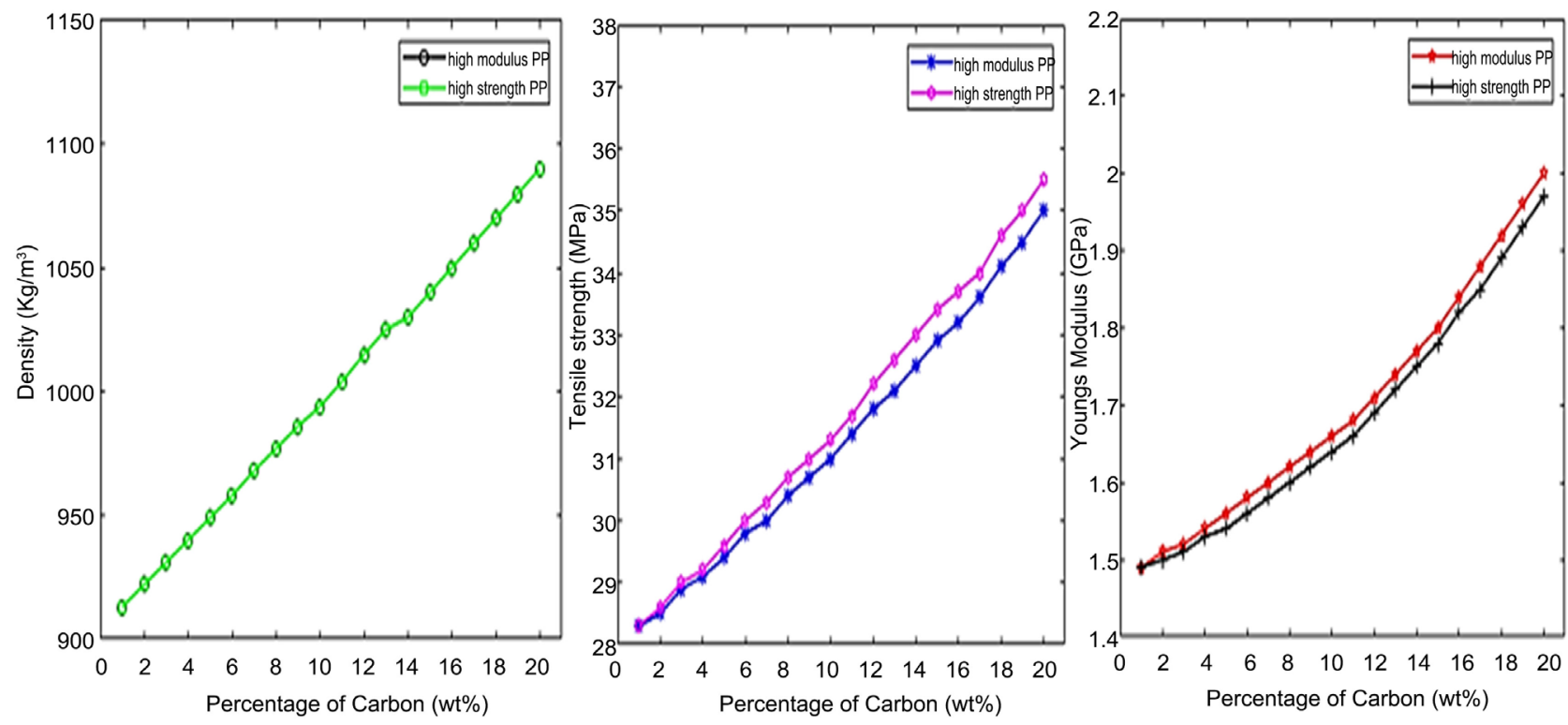

Figure 3. Simulated results for physical and mechanical properties of high modulus and high strength CFR-Recycled PP filament in varying composition up to $20 \mathrm{wt} \%$. 
Table 4. Simulated and experimental results of CFR-Recycled PP filament with 14 wt\% carbon fiber.

\begin{tabular}{ccc}
\hline Parameter & Simulated result & Experimental result \\
\hline Density $\left(\mathrm{Kg} / \mathrm{m}^{3}\right)$ & 1020 & - \\
Young's Modulus $(\mathrm{MPa})$ & 1590 & 1413 \\
Tensile strength $(\mathrm{MPa})$ & 26.1 & 24.2 \\
\hline
\end{tabular}

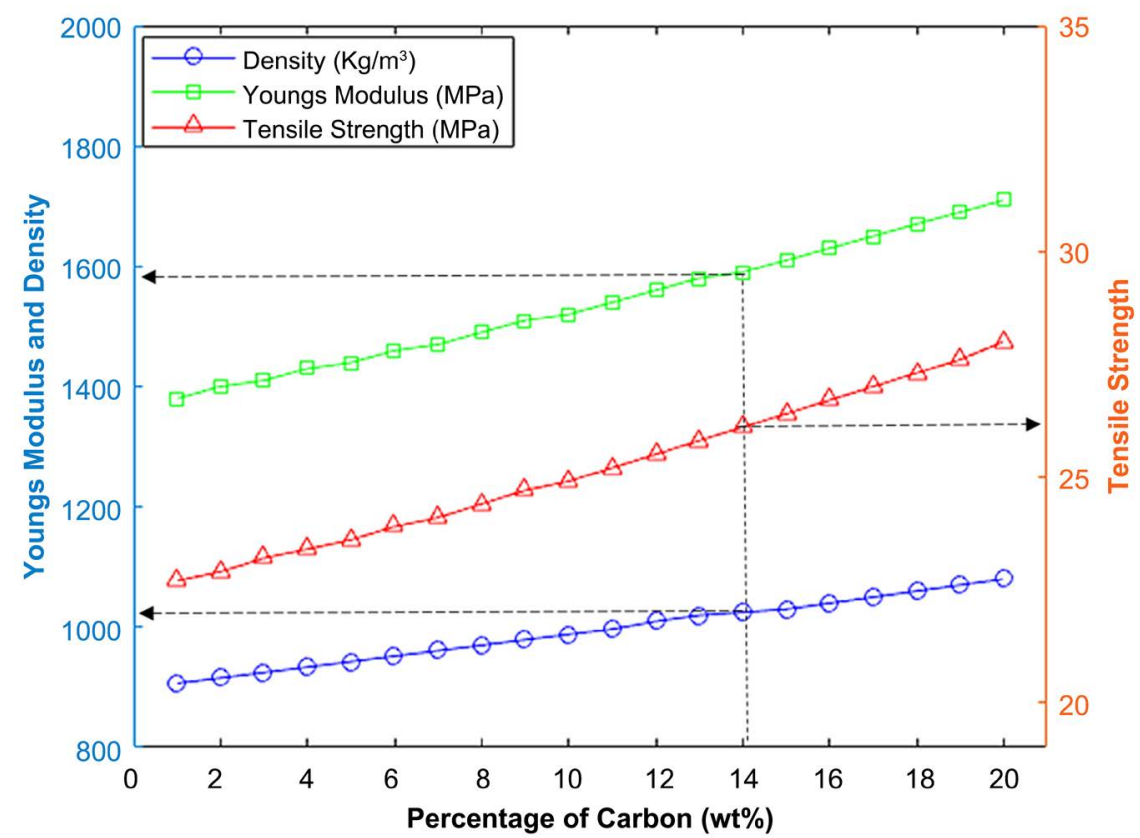

Figure 4. Simulated results for physical and mechanical properties of high modulus CFR-Recycled PP filament in varying composition up to $20 \mathrm{wt} \%$.

rollers as it passed through the water-cooled bath. The speed of this set of rollers (see Table 2) was adjusted until the filament diameter was of workable tolerance on the printer. The resulting filaments from recycled PP and CFR-recycled PP showed constant filament feed behavior during the printing process though they were slightly oval with dimension tolerance of $2.62( \pm 0.1) \times 2.82( \pm 0.1) \mathrm{mm}$ and $2.65( \pm 0.1) \times 2.75( \pm 0.1) \mathrm{mm}$ respectively. The fabricated filament was then passed through the filament storage rollers and later wound on a spooler at a controlled speed of $150 \mathrm{~mm} / \mathrm{s}$. Although the single screw extruder speed could be set to run up to $50 \mathrm{rpm}$, the filament extruded was too oval with minimum and maximum diameters of $2.22( \pm 0.1)$ and $2.82( \pm 0.1) \mathrm{mm}$, respectively.

SEM images also show that high extruder speeds yielded filaments that had a centreline cavity possibly due to the limited time given for the pellets to melt and mix well in the barrel, as shown in Figure 5(a). This was the main reason for setting and running the screw speed in the range of (13 - 20) rpm that resulted in filaments without the centreline cavity as shown in Figure 5(b). Scattered internal pores were also observed in the cross-sectional area of the CFR-recycled PP filaments, as shown in Figure 6(a) and Figure 6(c). Additionally, to ensure 


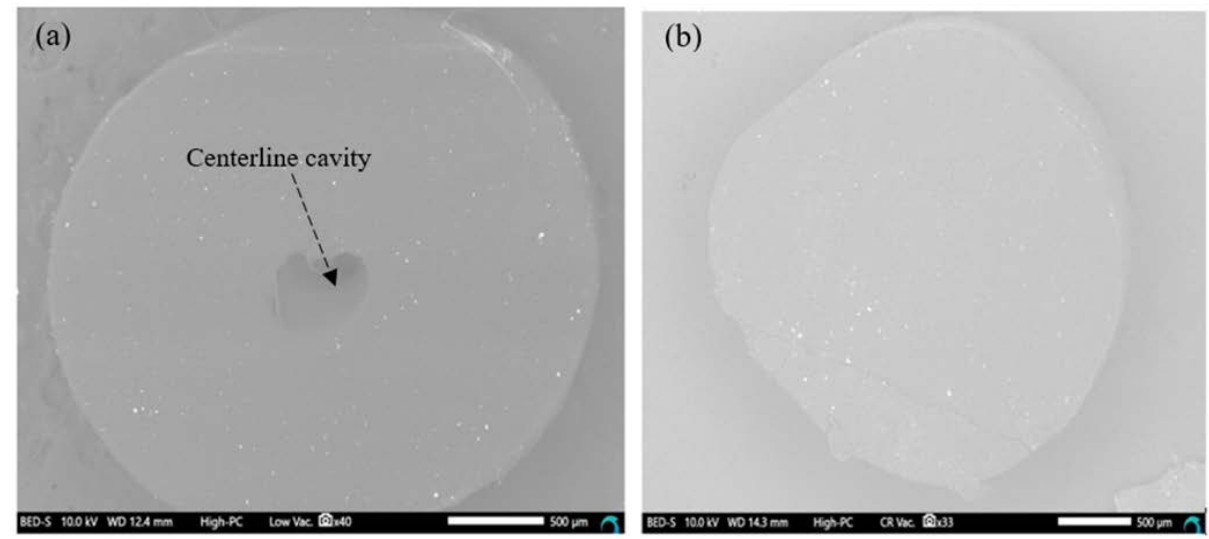

Figure 5. SEM images showing the microstructure of the extruded filaments of recycled PP with a centreline cavity after extrusion at 21 - 50 rpm screw speeds (a) and without the centreline cavity after extrusion at lower screw speeds of 13 - $20 \mathrm{rpm}$ (b).
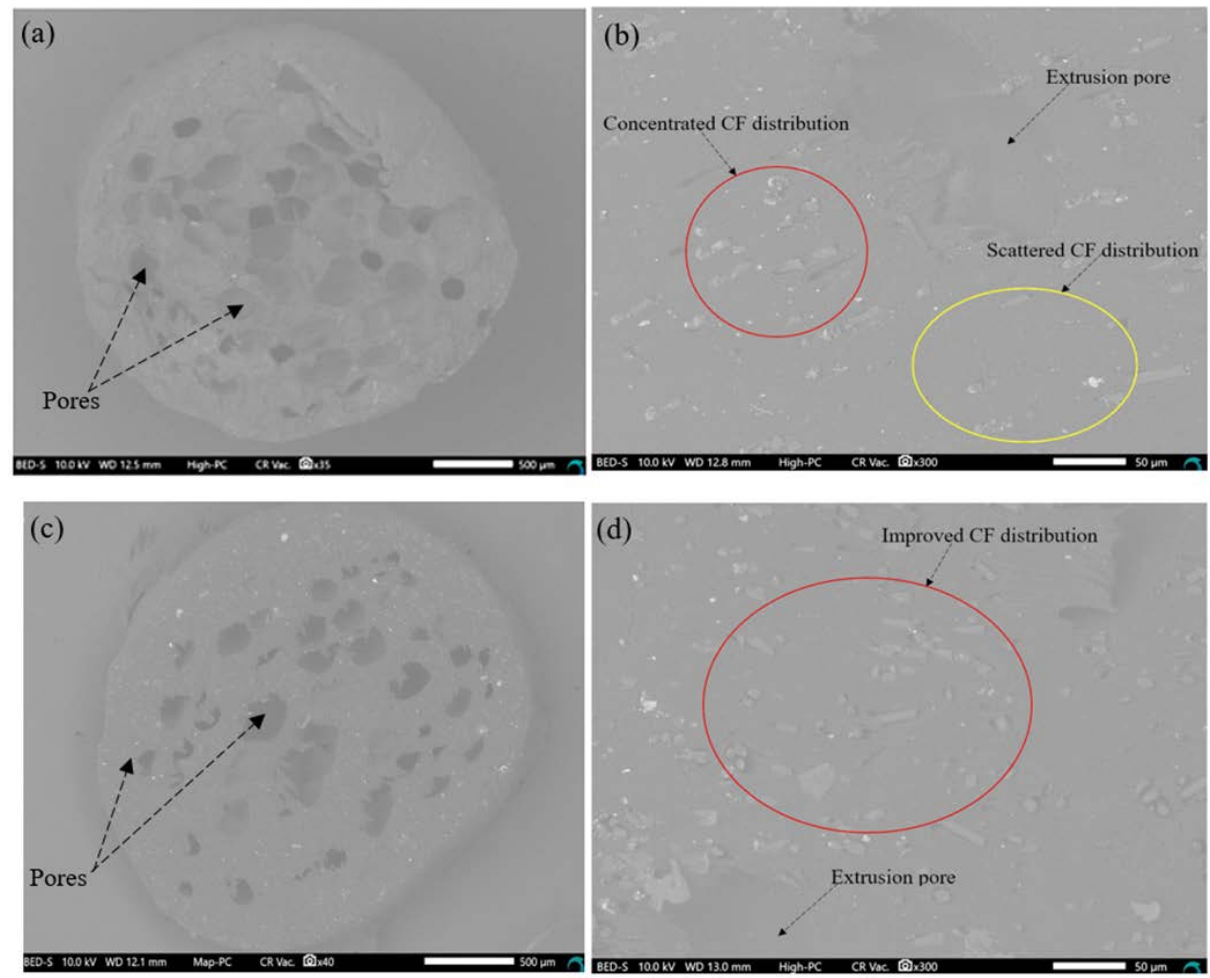

Figure 6. SEM images showing the microstructure of CFR-recycled PP filaments after the first extrusion stage (a) and the second extrusion (c). Inhomogeneous carbon fibre distribution is observed after the first filament extrusion as shown in (b) and improved carbon fibre distribution is shown in (d) after the second filament extrusion.

uniform mixing and distribution of the carbon fibre within the PP matrix, the composite filament was extruded in two stages. After the first extrusion, the composite filament was cut into small pieces by a pair of scissors and fed into the extruder for the second extrusion. This two-stage composite filament extrusion resulted in a better distribution of the carbon fibres in the PP matrix as shown in Figure 6(d) as compared to Figure 6(b). 


\subsection{Fracture Interface Observations of Samples Printed Using Recycled PP and CF/Recycled PP Filaments}

The fracture surfaces of tensile tested specimens show porosity and interlayer cavities as shown in Figure 7. The interlayer cavities are due to the physical gaps between each layer that were not completely filled during fused fabrication. The effect of reinforcing with short carbon fiber also resulted in the generation of extrusion pores in the composite filament as already described in Figure 6. These pores in the filaments are not eliminated during $3 \mathrm{D}$ printing as observed in the fracture surface of CFR-recycled PP specimens in Figure 7(d). Each printed layer and the adjacent raster layers of CFR-recycled PP specimens exhibited more and larger interlayer gaps and therefore significant reduction in the interfacial contact area between the printed layers. This explains the slight reduction in the mechanical properties for the composite filament and 3D printed parts compared to the simulated results (see Table 4). As shown in Figure 7(d), a more significant amount of carbon fibre ruptured at the fractured surfaces of the CFR-recycled PP specimens compared to the fewer ones that were pulled out. This indicates an excellent interfacial adhesion and tensile load transfer between the thermoplastic matrix and the reinforcing carbon fibre.

\subsection{Tensile Properties Recycled PP and CFR-Recycled PP}

Figure 8(a) shows the tensile strength of both filaments and 3D printed specimens manufactured using recycled PP and CFR-recycled PP. It can be seen that
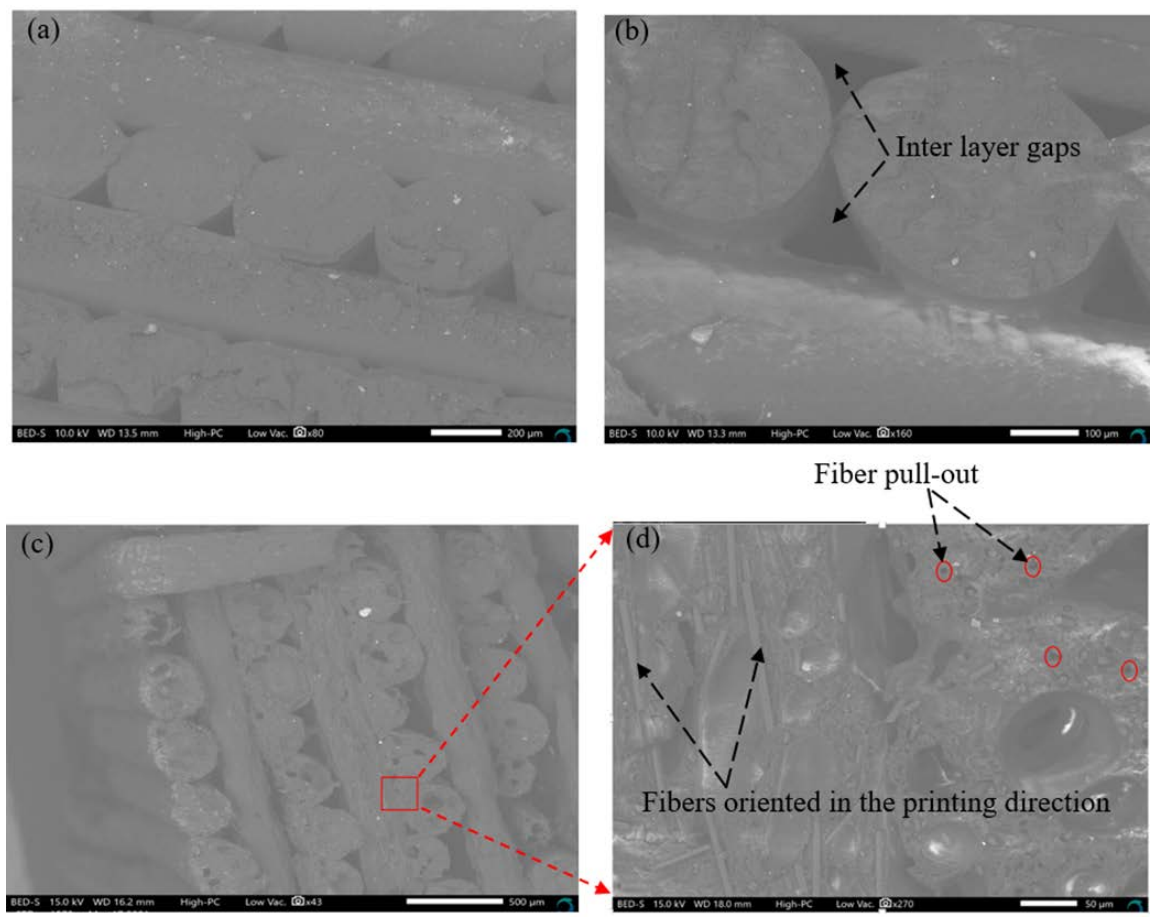

Figure 7. SEM images of tensile fracture surfaces for 3D printed specimens using recycled PP filament (a) and (b), CFR-recycled PP filament (c) and (d) showing inter-layer gaps, porosity, fibre pull-outs and general alignment of fibres in print direction. 


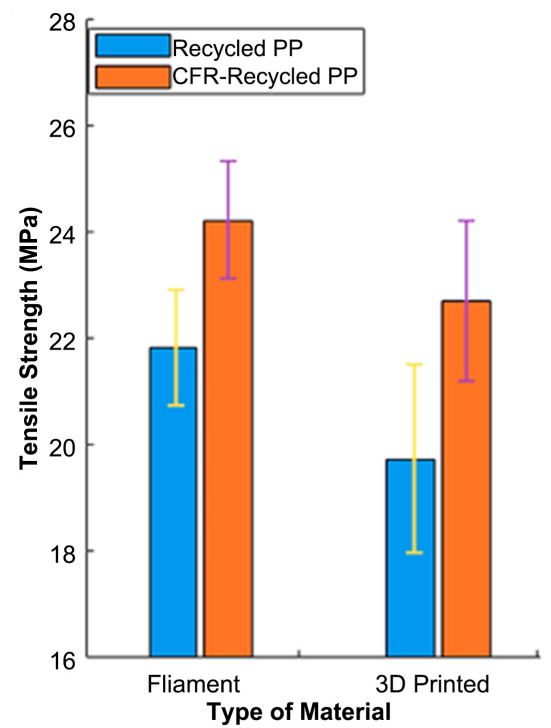

(a)

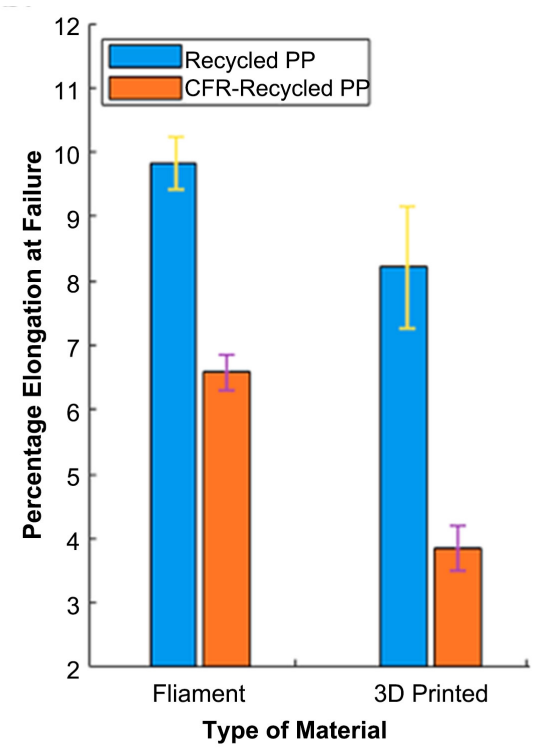

(b)

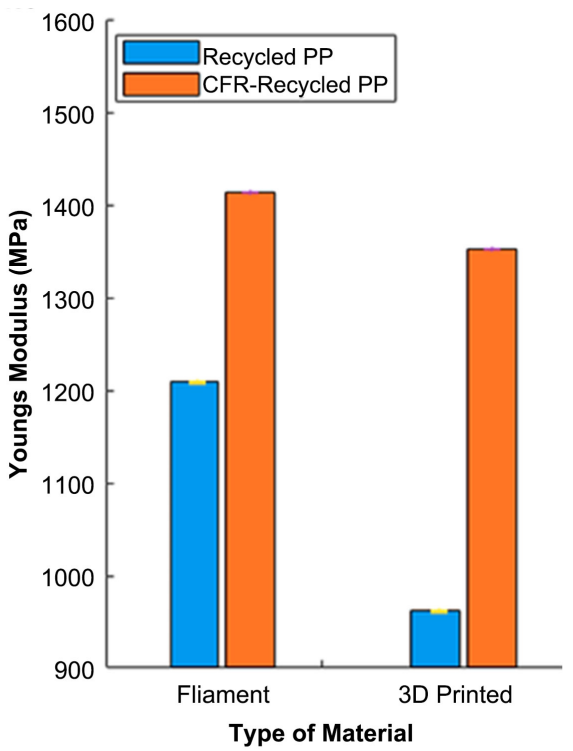

(c)

Figure 8. Tensile strength (a), percentage elongation at failure (b), and Young's Modulus (c) of recycled PP and CFR-recycled PP filaments compared to $3 \mathrm{D}$ printed specimens using the filaments.

the addition of short carbon fibres, with an average length of $200 \mathrm{um}$, to recycled PP increased the tensile strength of the filaments from 21.82 MPa to 24.22 MPa. Jiang et al. [23] reported the highest increase in tensile strength of $48.2 \%$ for $3 \mathrm{D}$ printed samples after addition of up to $16.8 \mathrm{wt} \%$ short carbon fibres ( 100 um average length) to ABS, PLA, PETG (polyethylele terephthalate glycol), and amphora polymers. However, when the recycled PP and CFR-recycled PP filaments were used for 3D printing of specimens, the tensile strengths reduced from 21.82 MPa and 24.22 MPa to 19.72 MPa and 22.70 MPa, respectively. This reduction is attributed to the existence of the inter-layer porosity and the anisotropic mechanical behavior typical in additive manufacturing since the tensile test samples were all printed at raster angles $[0,90]$. The improvement in mechanical strength of 3D printed samples using composite polymers brought by carbon fibre reinforcement is countered to a certain extent by the poor interfacial bonding between the 3D printed layers [4].

The percentage elongation at failure for both the filaments and 3D printed samples is shown in Figure 8(b). The value obtained for recycled PP filaments was $9.83 \%$ which reduced to $8.21 \%$ when the filament was used to $3 \mathrm{D}$ print samples. For the CFR-recycled PP filaments, the percentage elongation at failure was $6.58 \%$ which reduced to $3.84 \%$ for the $3 \mathrm{D}$ printed samples. The reduction of the percentage elongation at failure can be attributed to the addition of the brittle high modulus short carbon fibre. These fibres remained oriented in the printing direction as shown in Figure 6(d), similar to what Ferreira et al. [24] observed for 3D printed PLA reinforced with $15 \mathrm{wt} \%$ short carbon fibres.

The effect of short carbon fibre reinforcement on the Young's modulus is shown in Figure 8(c). The addition $14 \mathrm{wt} \%$ carbon fibre increased the modulus 
of the filaments from 1208.6 MPa to $1412.7 \mathrm{MPa}$, while the 3D printed specimens exhibited reduced modulus values of 961.5 MPa and 1352.3 MPa, respectively. When printing using both filaments, the values of Young's modulus reduced by $4.3 \%$ for CFR-recycled PP and $20.5 \%$ for recycled PP. This slight reduction in values for composite filament may be attributed to interlayer cavities and property anisotropy of the 3D printed specimens.

\section{Conclusions}

The microstructure and mechanical properties of extruded recycled polypropylene filaments without and with $14 \mathrm{wt} \%$ carbon fibre reinforcement were investigated. These filaments were subsequently used to $3 \mathrm{D}$ print specimens using fused filament fabrication to assess their suitability for additive manufacturing of commercial quality components. The following conclusions can be drawn from the results:

1) Recycled polypropylene filaments extruded at high screw speeds of above $20 \mathrm{rpm}$ exhibit a centreline cavity. However, this cavity is eliminated when extrusion is carried out at screw speeds below $20 \mathrm{rpm}$.

2) Recycled polypropylene filaments with $14 \mathrm{wt} \%$ short carbon fibre reinforcement exhibit pores that are dispersed throughout the microstructure of the filament. A two-stage extrusion improves the spatial distribution of carbon fibre reinforcement but does not reduce the pores.

3) Addition of $14 \mathrm{wt} \%$ carbon fibre reinforcement increases the tensile strength of the filaments from 21.82 MPa to 24.22 MPa and their Young's modulus from 1208.6 MPa to $1412.7 \mathrm{MPa}$. The elongation to failure is however reduced from $9.83 \%$ to $6.58 \%$.

4) Interlayer cavities were observed in $3 \mathrm{D}$ printed specimens. These cavities were larger for specimens printed from $14 \mathrm{wt} \%$ carbon fibre reinforced recycled polypropylene as compared with those printed from unreinforced filaments.

5) The tensile strength and Young's modulus of $3 \mathrm{D}$ printed specimens using $14 \mathrm{wt} \%$ carbon fibre reinforcement filaments are higher (22.70 MPa and 1352.3 $\mathrm{MPa}$, respectively) compared to the tensile strength and Young's modulus of 3D printed specimens without reinforcement (19.72 MPa and 961.5 MPa, respectively). The elongation to failure is however reduced from $8.21 \%$ for specimens without reinforcement to $3.84 \%$ for specimens with reinforcement.

6) The mechanical properties of the $3 \mathrm{D}$ printed specimens are lower than those of the corresponding filaments used to $3 \mathrm{D}$ print for the specimens. This is attributed to interlayer cavities observed in $3 \mathrm{D}$ printed specimens and property anisotropy associated with fused filament fabrication.

\section{Acknowledgements}

The authors acknowledge the financial support provided by the Pan African University through the Institute of Basic Sciences, Technology \& Innovation (PAUSTI) and Japan International Cooperation Agency (JICA). 


\section{Conflicts of Interest}

The authors declare no conflicts of interest regarding the publication of this paper.

\section{References}

[1] Tahmasebinia, F., et al. (2018) Three-Dimensional Printing Using Recycled High-Density Polyethylene: Technological Challenges and Future Directions for Construction. Buildings, 8, 165. https://doi.org/10.3390/buildings8110165

[2] Sodeifian, G., Ghaseminejad, S. and Yousefi, A.A. (2019) Preparation of Polypropylene/Short Glass Fiber Composite as Fused Deposition Modeling (FDM) Filament. Results in Physics, 12, 205-222. https://doi.org/10.1016/j.rinp.2018.11.065

[3] Gupta, A., Fidan, I., Hasanov, S. and Nasirov, A. (2020) Processing, Mechanical Characterization, and Micrography of 3D-Printed Short Carbon Fiber Reinforced Polycarbonate Polymer Matrix Composite Material. The International Journal of Advanced Manufacturing Technology, 107, 3185-3205. https://doi.org/10.1007/s00170-020-05195-Z

[4] Wang, X., Jiang, M., Zhou, Z., Gou, J. and Hui, D. (2017) 3D Printing of Polymer Matrix Composites: A Review and Prospective. Composites Part B: Engineering, 110, 442-458. https://doi.org/10.1016/j.compositesb.2016.11.034

[5] Novakova-Marcincinova, L. and Kuric, I. (2012) Basic and Advanced Materials for Fused Deposition Modeling Rapid Prototyping Technology. Manuf. and Ind. Eng, 11, 1338-6549.

[6] Nyiranzeyimana, G., Mutua, J.M., Mose, B.R. and Mbuya, T.O. (2021) Optimization of Process Parameters in Fused Deposition Modelling of Thermoplastics: A Review. Materialwissenschaft und Werkstofftechnik, 52, 682-694. https://doi.org/10.1002/mawe.202000193

[7] Arivazhagan, A. and Masood, S.H. (2012) Dynamic Mechanical Properties of ABS Material Processed by Fused Deposition Modelling. International Journal of Engineering Research and Applications, 2, 2009-2014.

[8] Spoerk, M., Holzer, C. and Gonzalez-Gutierrez J. (2020) Material Extrusion-Based Additive Manufacturing of Polypropylene: A Review on How to Improve Dimensional Inaccuracy and Warpage. Journal of Applied Polymer Science, 137, Article ID: 48545 . https://doi.org/10.1002/app.48545

[9] Busch, S.F., Weidenbach, M., Fey, M., Schäfer, F., Probst, T. and Koch, M. (2014) Optical Properties of 3D Printable Plastics in the THz Regime and Their Application for 3D Printed THz Optics. Journal of Infrared, Millimeter, and Terahertz Waves, 35, 993-997. https://doi.org/10.1007/s10762-014-0113-9

[10] Cruz, F., Lanza, S., Boudaoud, H., Hoppe, S. and Camargo, M. (2012) Polymer Recycling and Additive Manufacturing in an Open Source context: Optimization of Processes and Methods. Solid Freeform Fabrication, 1591-1600.

[11] Cruz Sanchez, F.A., Boudaoud, H., Hoppe, S. and Camargo, M. (2017) Polymer Recycling in an Open-Source Additive Manufacturing Context: Mechanical Issues. Additive Manufacturing, 17, 87-105. https://doi.org/10.1016/j.addma.2017.05.013

[12] Mulder, M.L., Glover, A.G. and Pearce, J.M. (2014) Life Cycle Analysis of Distributed Recycling of Post-consumer High Density Polyethylene for 3-D Printing Filament. Journal of Cleaner Production, 70, 90-96.

[13] Spoerk, M., Arbeiter, F., Raguž, I., Holzer, C. and Gonzalez-Gutierrez, J. (2019) 
Mechanical Recyclability of Polypropylene Composites Produced by Material Extrusion-Based Additive Manufacturing. Polymers (Basel), 11, 1318. https://doi.org/10.3390/polym11081318

[14] Zander, N.E., Gillan, M., Burckhard, Z. and Gardea, F. (2019) Recycled Polypropylene Blends as Novel 3D Printing Materials. Additive Manufacturing, 25, 122-130. https://doi.org/10.1016/j.addma.2018.11.009

[15] Naranjo-Lozada, J., Ahuett-Garza, H., Orta-Castañón, P., Verbeeten, W.M.H. and Sáiz-González, D. (2019) Tensile Properties and Failure Behavior of Chopped and Continuous Carbon Fiber Composites Produced by Additive Manufacturing. Additive Manufacturing, 26, 227-241. https://doi.org/10.1016/j.addma.2018.12.020

[16] Goh, G.D., Yap, Y.L., Agarwala, S. and Yeong, W.Y. (2019) Recent Progress in Additive Manufacturing of Fiber Reinforced Polymer Composite. Advanced Materials Technologies, 4, 1-22. https://doi.org/10.1002/admt.201800271

[17] Ivey, M., Melenka, G.W., Carey, J.P. and Ayranci, C. (2017) Characterizing Short-Fiber-Reinforced Composites Produced Using Additive Manufacturing. Advanced Manufacturing: Polymer \& Composites Science, 3, 81-91. https://doi.org/10.1080/20550340.2017.1341125

[18] Ning, F., Cong, W., Qiu, J., Wei, J. and Wang, S. (2015) Additive Manufacturing of Carbon Fiber Reinforced Thermoplastic Composites Using Fused Deposition Modeling. Composites Part B: Engineering, 80, 369-378. https://doi.org/10.1016/j.compositesb.2015.06.013

[19] Mori, K.I., Maeno, T. and Nakagawa, Y. (2014) Dieless Forming of Carbon Fibre Reinforced Plastic Parts Using 3D Printer. Procedia Engineering, 81, 1595-1600. https://doi.org/10.1016/j.proeng.2014.10.196

[20] Materials, P., Materials, E.I., Matrix, P., Materials, C. and Specimens, P. (2006) Standard Test Method for Tensile Properties of Plastics 1. 1-15.

[21] Schirmeister, C.G., Hees, T., Licht, E.H. and Mülhaupt, R. (2019) 3D Printing of High Density Polyethylene by Fused Filament Fabrication. Additive Manufacturing, 28, 152-159. https://doi.org/10.1016/j.addma.2019.05.003

[22] Ultimaker (2018) Technical Data Sheet PP Filament.

[23] Jiang, D. and Smith, D.E. (2017) Anisotropic Mechanical Properties of Oriented Carbon Fiber Filled Polymer Composites Produced with Fused Filament Fabrication. Additive Manufacturing, 18, 84-94. https://doi.org/10.1016/j.addma.2017.08.006

[24] Ferreira, R.T.L., Amatte, I.C., Dutra, T.A. and Bürger, D. (2017) Experimental Characterization and Micrography of 3D Printed PLA and PLA Reinforced with Short Carbon Fibers. Composites Part B: Engineering, 124, 88-100. https://doi.org/10.1016/j.compositesb.2017.05.013 